\title{
Muscle weakness, cognitive impairment and their interaction on altered balance in elderly outpatients: results from the TRIP observational study
}

This article was published in the following Dove Press journal:

Clinical Interventions in Aging

\author{
Fulvio Lauretani ${ }^{1,2}$ \\ Marcello Maggio ${ }^{1,2}$ \\ Andrea Ticinesi ${ }^{1,2}$ \\ Claudio Tana ${ }^{1,2}$ \\ Beatrice Prati ${ }^{1,2}$ \\ Luciano Gionti ${ }^{1,2}$ \\ Antonio Nouvenne ${ }^{1,2}$ \\ Tiziana Meschil,2 \\ 'Department of Medicine and \\ Surgery, University of Parma, Parma, \\ Italy; ${ }^{2}$ Internal Medicine and Critical \\ Subacute Care Unit, Geriatric \\ Rehabilitation Department, University \\ Hospital of Parma, Parma, Italy
}

Correspondence: Claudio Tana Internal Medicine and Critical Subacute Care Unit, Geriatric-Rehabilitation Department, University Hospital, Via Gramsci I4, 43I 25 Parma, Italy Tel +3952I 704316 Email ctana@ao.pr.it
Background: The determinants of altered balance in older subjects and, particularly, the contribution of muscle strength and cognitive impairment are still uncertain. We hypothesized that both these conditions and their interactions could affect balance in older persons. To address this hypothesis, we studied the cross-sectional association between handgrip strength and balance performance in subjects with and without cognitive impairment.

Methods: Two hundred and sixty-three elderly outpatients (104 men and 159 women, aged $81.44 \pm 7.36$ years) were evaluated for the comprehensive geriatric assessment. The patients were enrolled from the cross-sectional observational Traumatic Risk Identikit Parma study that was aimed at identifying risk factors for falling in older outpatients.

Results: Balance deficit, defined as an inability to maintain tandem position for at least 10 seconds, was present in 185 patients $(70.34 \%)$. The mean mini-mental state examination score was $20.79 \pm 6.5$, the median short physical performance battery score was 5.0 and the mean grip strength was $18.54 \pm 9.14 \mathrm{~kg}$. After dividing the subjects into four categories according to the presence of low grip strength and/or cognitive impairment, we found that the mini nutritional assessment short-form scale score was negatively and independently $(\beta$ : $-0.02 \pm$ $0.01 ; p=0.04$ ) associated with balance deficit in the group with low grip strength and cognitive impairment. In the other three categories, grip strength was negatively and significantly associated with balance deficit.

Conclusion: The loss of muscle strength, presence of cognitive impairment and their interaction, influenced probably by malnutrition, could affect balance in older persons.

Keywords: muscle strength, cognitive impairment, balance, malnutrition, older persons

\section{Background}

Falls are potentially catastrophic events which predominantly occur in elderly subjects. In populations older than 65 years, almost one-third of community-dwelling older subjects fall at least once per year, and this prevalence increases with aging. ${ }^{1}$ Around $10 \%$ of falls causes hip fracture ${ }^{2}$ and half of these patients became permanently disabled. The real efficacy of falls prevention to reduce fractures is still debated, ${ }^{3}$ and this is related to a different fall risk profile of older people and also to the type of intervention program carried out. ${ }^{4}$

There is no international consensus to describe the fall risk profile of older subjects, although it is well understood that older people at higher risk of future falls are those with previous falls, who have reduced walking speed, fear of falling and pathological 
changes in muscle function or balance problems. ${ }^{5,6}$ The fall risk profile is also dependent on the presence of surrounding environmental obstacles, such as unsafe walking surfaces and other factors, such as cognitive impairment, which may be associated with loss of attention, executive dysfunction and an increase of rapid and afinalistic movements. Although the decline in balance, gait and muscle function increases the risk of falling in older persons, the relationship is probably exponential and dependent on to the plethora of co-impairments and comorbidity. ${ }^{7}$

Mobility difficulties in the elderly are significant predictors of subsequent falls, ${ }^{1}$ which can lead to disability, institutionalization and mortality. ${ }^{8,9}$ Balance plays a critical role in the maintenance of postural stability, thereby affecting mobility. ${ }^{10}$ Advancing age is thought to impair the ability to control balance while walking, ${ }^{11}$ and evidence suggests that age-associated changes particularly in muscle strength and cognitive performance may be responsible for the altered or impaired balance control experienced by older adults. ${ }^{12}$

A combined and simultaneous evaluation of muscle strength in the lower limbs and cognitive performance could more appropriately define the effects of the mixed alteration of age and diseases on balance in older persons regarding the separate aspects of reduced muscle strength or malnutrition or presence of cognitive impairment. ${ }^{13-15}$ In this study, a significant interaction between muscle strength and cognitive function in determining balance alteration can be hypothesized.

However, there are few data about this association in the older population. In this study, we hypothesized an interaction between muscle strength and cognitive function, particularly in patients with an impairment of both conditions, influencing balance and therefore the risk of falls.

To address this hypothesis, we studied the cross-sectional association between grip strength and balance performance in elderly patients who were classified according to the presence of muscle weakness and/or cognitive impairment.

\section{Methods}

Outpatients presented to the Cognitive and Motor Center of the Geriatric-Rehabilitation Department of Parma, Italy, were evaluated for the comprehensive geriatric assessment (CGA), as requested by their own general practitioner or after discharge from Medicine and Geriatric-Rehabilitation Department Units. We selected 263 subjects older than 65 years (104 men and 159 women, $81.44 \pm 7.36$, mean age \pm SD) between September and December 2017 from the prospective observational Traumatic Risk Identikit Parma (TRIP) study.
This is a cross-sectional observational study, with the primary aim of identifying risk factors for reported falls in the years before the baseline evaluation. Exclusion criteria were as follows: 1) reported severe ADL and mobility disability at baseline, 2) terminal health condition and 3) unwillingness to provide written informed consent.

All patients received a standardized medical visit. ${ }^{16}$ The CGA included information of drugs and comorbidities ${ }^{17}$ as well as evaluation of body mass index (BMI) and muscle and cognitive performance, such as handgrip strength and the short physical performance battery (SPPB), mini-mental state examination (MMSE), clock drawing test (CDT) and mini nutritional assessment short-form (MNA-SF) scales. Depression was evaluated using the geriatric depression scale (GDS), and when the diagnosis of dementia was unclear, amyloid positron emission tomography (PET) was prescribed after computed tomography (CT)-brain evaluation. ${ }^{18}$ Balance deficit was defined as an inability to maintain tandem position (one foot in front of the other) for at least 10 seconds. This evaluation was part of the SPPB total score. Patients who reported a history of falls in the year before evaluation were classified as fallers.

According to our initial hypothesis, we then divided the population into four groups: 1) low grip strength and cognitive impairment (grip strength lower than $26 \mathrm{~kg}$ for men and $16 \mathrm{~kg}$ for women ${ }^{19}$ and MMSE lower than 24/30, indicating cognitive impairment); 2) low grip strength without cognitive impairment (grip strength lower than $26 \mathrm{~kg}$ for men and $16 \mathrm{~kg}$ for women and MMSE higher than 24/30); 3) cognitive impairment with normal grip strength (MMSE lower than 24/30 and grip strength higher than $25 \mathrm{~kg}$ for men and $15 \mathrm{~kg}$ for women) and 4) normal grip strength without cognitive impairment.

The data were treated in agreement with the Italian law to guarantee privacy. A detailed informed consent was signed by all study participants. We applied good clinical practice to detect causes of falls and different types of dementia in older persons. The ethics committee of the University Hospital of Parma approved the study (ID 17262). All experiments were performed in accordance with the current relevant guidelines and regulations.

\section{Statistical analyses}

Data are reported as mean and standard deviation or median and IQR or percentages. Factors associated with balance deficit were tested with an age- and sex-adjusted Pearson's correlation analysis. A multivariate regression analysis was used to test the relationship between balance deficit and the cognitive and physical performance of participants, also 
considering an interaction term "MMSE*muscle strength" as an independent variable.

A multivariate regression analysis was performed for each group to test the relationship between balance deficit and cognitive and physical performance of participants. Statistical significance was defined at a $p$-value $<0.05$. The statistical processing was carried out using SAS 8.2 software (C) SAS Institute Inc.,Cary, NC, USA).

\section{Results}

The demographic and clinical characteristics of the study population are given in Table 1. Among 263 participants, 114 were categorized as "cognitively impaired with low grip strength", 38 as "low grip strength with normal cognition", 48 as "cognitively impaired with normal grip strength" and 63 as "normal grip strength with normal cognition". Balance deficit was detected in 185 patients (70.34\%). The mean MMSE score was $20.79 \pm 6.5$, the median SPPB score was $5.0,{ }^{2-11}$ while the mean grip strength was $18.54 \pm 9.14 \mathrm{~kg}$. One hundred and ten subjects $(42 \%)$ reported at least one fall in the year before the evaluation and were thus considered fallers.

Table 2A shows the correlation between balance deficit and characteristics of the subjects obtained via the CGA.

Table I Characteristics of the study population ( $\mathrm{N}=263)$

\begin{tabular}{lll}
\hline & Mean & SD \\
\hline Age (years) & 81.44 & 7.36 \\
Female (n, \%) & 159 & 60.46 \\
Balance deficit* (n, \%) & 185 & 70.34 \\
Low grip strength** (n, \%) & 152 & 57.8 \\
Cognitive impairment*** (n, \%) & 162 & 61.6 \\
Low grip strength with cognitive impairment (n, \%) & 114 & 43.35 \\
Low grip strength without cognitive impairment & 38 & 14.45 \\
(n, \%) & & \\
Cognitive impairment with normal grip strength & 48 & 18.25 \\
(n, \%) & & \\
Normal grip strength without cognitive & 63 & 23.95 \\
impairment (n, \%) & & \\
Falls in the year before evaluation (n, \%) & 110 & 42.0 \\
Number of drugs (<5) (n, \%) & 94 & 35.7 \\
SPPB score (median, IQR) & 5 & $2-11$ \\
BMI (kg/m²) & 26.64 & 5.14 \\
MMSE score & 20.79 & 6.50 \\
Grip strength (kg) & 18.54 & 9.14 \\
4m-WS (m/s) & 0.60 & $0.22-0.88$ \\
MNA-SF & 10.58 & 2.71 \\
GDS-I5 points & 3 & $1-5$ \\
\hline Nit & & \\
\hline
\end{tabular}

Notes: *Defined as inability to maintain Tandem position for at least 10 seconds. **Defined as grip strength below $26 \mathrm{~kg}$ in men and $16 \mathrm{~kg}$ in women. ***Defined as MMSE below 24. ${ }^{\S 4-m}$ walking speed usual pace.

Abbreviations: SPPB, short physical performance battery; IQR, interquartile range; BMI, body mass index; MMSE, mini-mental state examination; MNA-SF, mini nutritional assessment short-form; GDS, geriatric depression scale.
Table 2A Factors associated with balance deficit

\begin{tabular}{lll}
\hline & $\beta$ & $p$-value* \\
\hline Grip strength & -0.39 & $<0.0001$ \\
MMSE & -0.17 & 0.01 \\
BMI & 0.05 & 0.42 \\
MNA-SF & -0.25 & 0.0002 \\
Number of drugs & 0.19 & 0.005 \\
GDS & 0.20 & 0.16 \\
\hline
\end{tabular}

Note: *Age- and sex-adjusted Pearson's correlation analysis.

Abbreviations: MMSE, mini-mental state examination; BMI, body mass index; MNA-SF, mini nutritional assessment short-form; GDS, geriatric depression scale.

In the age- and sex-adjusted analysis, grip strength, MMSE and MNA-SF were negatively associated and the number of drugs was positively associated with balance deficit.

Figure 1 shows the close association between grip strength and MMSE $(p<0.0001)$, and given the indipendent association of each parameter with balance deficit, we addressed the hypothetical presence of a relationship between the interaction term muscle and cognition and balance deficit. We found an independent relationship of the interaction "Grip strength*MMSE" and balance deficit ( $p=0.02$, Table 2B). After the division of the population into four categories, based on the presence of muscle and cognitive impairment (Table 1), we found that almost all subjects with both impairments had a balance deficit (91\%), $70 \%$ of subjects with low grip strength and $61 \%$ of subjects with cognitive impairment had balance deficit, and only $38 \%$ of patients without muscle and cognitive impairment showed a balance deficit (Figure 2). We also reported the age- and sex-association between grip strength, MMSE and balance deficit among the four categories (Table 3). We found that MMSE was negatively associated

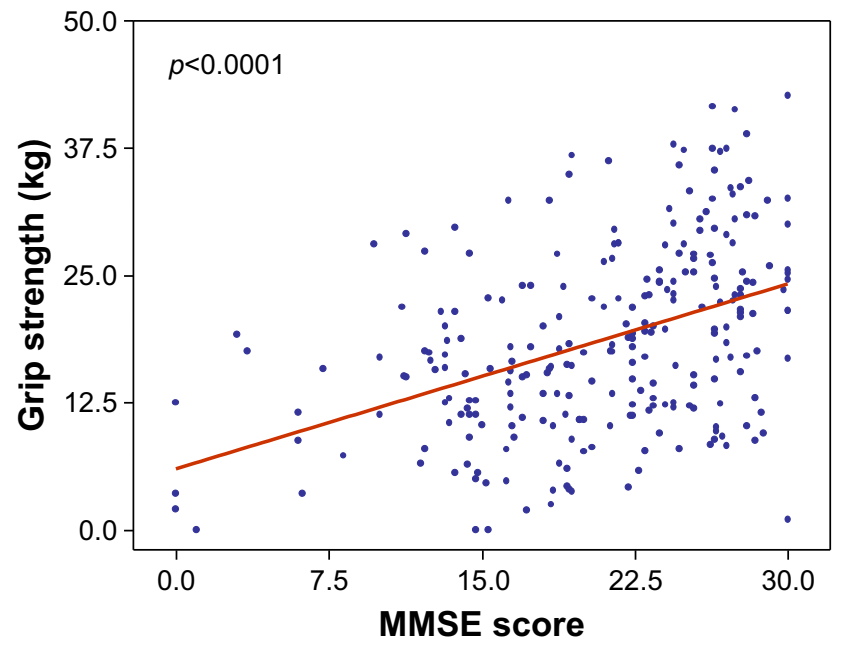

Figure I Association between grip strength and MMSE $(p<0.000$ I). Abbreviation: MMSE, mini-mental state examination. 
Table 2B Association between muscle strength, cognitive performance and their interaction term on balance deficit

\begin{tabular}{llll}
\hline & \multicolumn{3}{l}{ Balance deficit } \\
\cline { 2 - 4 } & $\boldsymbol{\beta}$ & $\mathrm{SE}$ & $\boldsymbol{p}$-value \\
\hline Grip strength & -0.002 & 0.009 & 0.87 \\
MMSE & 0.009 & 0.007 & 0.25 \\
“Grip strength*MMSE” & -0.01 & 0.0004 & 0.02 \\
Age & 0.01 & 0.003 & 0.0007 \\
Sex (M versus F) & -0.02 & 0.06 & 0.69 \\
\hline
\end{tabular}

Abbreviations: SE, standard error; MMSE, mini-mental state examination; M, male; $\mathrm{F}$, female.

with balance deficit in those with low grip strength ( $p=0.04)$; in contrast, grip strength was negatively associated with balance deficit in those with cognitive impairment ( $p=0.02$ ).

Finally, we reported the multivariate regression analysis of factors independently associated with the balance deficit in the four categories of patients (Table 4).

In the first category (low grip strength and cognitive impairment), we found that MNA-SF, a marker of nutrition status, was negatively and independently associated with a balance deficit ( $p=0.04$ ). In the other three categories, grip strength was negatively associated with balance deficit.

\section{Discussion}

Our results suggest that grip strength is one of the main factors affecting balance in older persons. The data show that cognitive performance influences balance in older persons

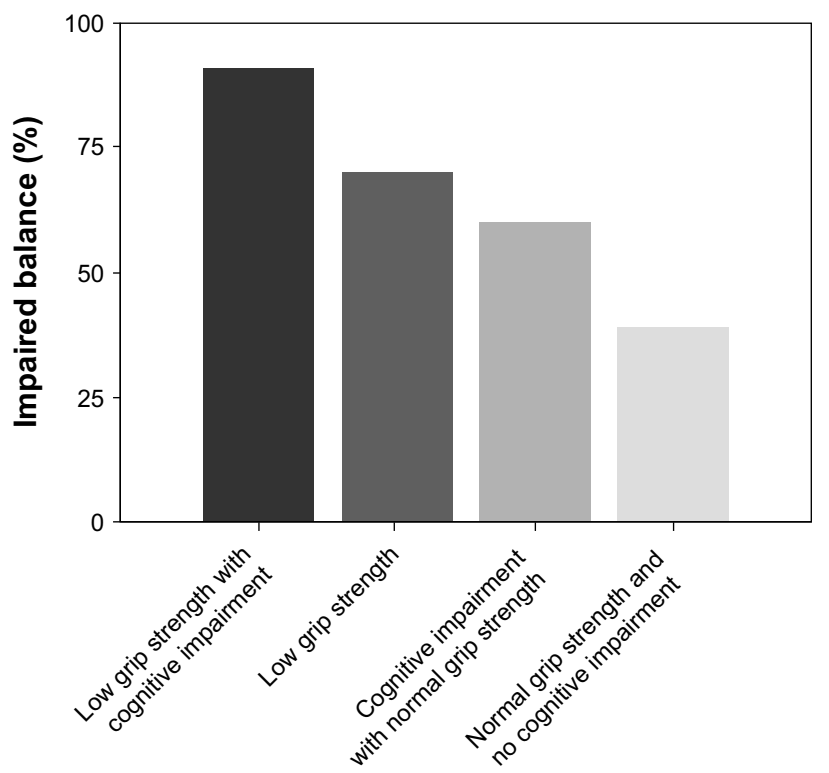

Figure 2 Relationship between cognitive impairment, grip strength and balance deficit: almost all patients with both impairments had a balance deficit (91\%), 70\% of those with low grip strength and $61 \%$ of those with cognitive impairment had a balance deficit, while only $38 \%$ of patients without muscle and cognitive impairment showed a balance deficit (Chi-square $p<0.0001$ ).
Table 3 Age- and sex-adjusted muscle strength and MMSE with the balance deficit among 4 categories

\begin{tabular}{lcc}
\hline Balance deficit & $\beta \pm \mathbf{S E}$ & $p$-value \\
\hline I. Low grip strength with cognitive impairment $(n=I \mid 4)$ & \\
MMSE & $-0.002 \pm 0.004$ & 0.73 \\
Grip strength & $-0.04 \pm 0.005$ & 0.46 \\
2. Low grip strength without cognitive impairment $(n=38)$ & \\
MMSE $\quad-0.08 \pm 0.3$ & 0.04 \\
Grip strength & $-0.0017 \pm 0.0049$ & 0.73 \\
3. Cognitive impairment with normal grip strength $(n=48)$ & \\
MMSE & $-0.004 \pm 0.014$ & 0.77 \\
Grip strength & $-0.05 \pm 0.02$ & 0.02 \\
4. Normal grip strength without cognitive impairment $(n=63)$ & \\
MMSE & $-0.04 \pm 0.03$ & 0.30 \\
Grip strength & $-0.01 \pm 0.01$ & 0.35 \\
\hline
\end{tabular}

Abbreviations: MMSE, mini-mental state examination; SE, standard error.

and also a significant interaction between these two factors could significantly accelerate balance deficit in advanced age. When poor grip strength and cognitive performance coexist, an evaluation of nutritional status is advisable, because balance could also be associated with malnutrition in patients with cognitive impairment and reduced muscle strength.

These results support the use of an integrated evaluation of the motoric and cognitive function, especially in older persons with a risk of falling. Both muscle strength and lower physical function assessment should be part of a cognitive evaluation in the presence or absence of cognitive impairment, surpassing the current separate evaluation of memory deficit and movement disorders in older persons. ${ }^{20}$ Based on these results, an evaluation of physical and cognitive performance should be promptly and simultaneously conducted in older persons when they are admitted to a geriatric setting in order to identify subjects at risk of falling. With this regard, "cross-road" tests able to simultaneously detect motoric and cognitive function are particularly useful. Stress-tests such as dual-task test, "walking by taking" or walking with obstacles, are good examples and allow the quantification of the risk of falls and potentially predict consequences that could be simple bruises, hip fractures or even head trauma with subsequent catastrophic and permanent disability.

The "fall" might be considered a hypothetical "crossroad" clinical event linking cognitive and motoric domains and is normally accompanied by subsequent fear of falling and impaired quality of life. ${ }^{21} \mathrm{~A}$ fall by older people could be considered an event related to the loss of homeostasis of many systems, for example, the presence of multiple cerebral vascular lesions could produce at the same time 
Table 4 Relationship between balance deficit and characteristics derived from the CGA among four categories

\begin{tabular}{|c|c|c|c|c|c|c|c|c|}
\hline & \multicolumn{2}{|c|}{$\begin{array}{l}\text { I. Low grip strength } \\
\text { with cognitive } \\
\text { impairment }\end{array}$} & \multicolumn{2}{|c|}{$\begin{array}{l}\text { 2. Low grip strength } \\
\text { without cognitive } \\
\text { impairment }\end{array}$} & \multicolumn{2}{|c|}{$\begin{array}{l}\text { 3. Cognitive impairment } \\
\text { with normal grip } \\
\text { strength }\end{array}$} & \multicolumn{2}{|c|}{$\begin{array}{l}\text { 4. Normal grip strength } \\
\text { without cognitive } \\
\text { impairment }\end{array}$} \\
\hline & $\beta \pm \mathrm{SE}$ & $p$-value & $\beta \pm \mathrm{SE}$ & $p$-value & $\beta \pm \mathrm{SE}$ & $\overline{p \text {-value }}$ & $\beta \pm \mathrm{SE}$ & $p$-value \\
\hline Age & $0.009 \pm 0.004$ & 0.05 & $0.001 \pm 0.009$ & 0.87 & $0.03 \pm 0.02$ & 0.05 & $0.03 \pm 0.009$ & 0.005 \\
\hline Sex & $-0.08 \pm 0.07$ & 0.11 & $-0.07 \pm 0.22$ & 0.75 & $0.03 \pm 0.34$ & 0.37 & $0.029 \pm 0.22$ & 0.87 \\
\hline MNA-SF & $-0.02 \pm 0.01$ & 0.04 & $-0.02 \pm 0.03$ & 0.16 & $0.027 \pm 0.037$ & 0.46 & $0.024 \pm 0.037$ & 0.52 \\
\hline Grip strength & $0.004 \pm 0.005$ & 0.47 & $-0.03 \pm 0.009$ & 0.01 & $-0.02 \pm 0.02$ & 0.001 & $-0.02 \pm 0.008$ & 0.01 \\
\hline MMSE & $-0.001 \pm 0.005$ & 0.83 & $-0.051 \pm 0.04$ & 0.22 & $-0.018 \pm 0.015$ & 0.23 & $-0.02 \pm 0.04$ & $0.5 \mathrm{I}$ \\
\hline Number of drugs & $-0.036 \pm 0.037$ & 0.34 & $0.17 \pm 0.09$ & 0.07 & $0.17 \pm 0.08$ & 0.04 & $0.042 \pm 0.064$ & 0.52 \\
\hline
\end{tabular}

Abbreviations: CGA, comprehensive geriatric assessment; SE, standard error; MNA-SF, mini nutritional assessment short-form; MMSE, mini-mental state examination.

a decline in cognitive performance, executive function deficits, walking speed reduction, loss of mechanism of balance compensation and, finally, a reduction of attention and reaction time. Moreover, Beauchet et $\mathrm{al}^{22}$ recently proposed a new syndrome, the motoric-cognitive syndrome, which includes subjective memory loss associated with low gait speed and reduced physical performance in older persons, where falls could be recognized as the first event that reveals this condition, with a subsequent list of scale-up assessments. 22

Muscle strength is particularly influenced by physical activity and resistance training exercise which can be considered a therapeutic strategy in many older subjects who have reduced cognitive and physical performance. ${ }^{23}$ In both primary and secondary sarcopenia with low muscle strength, resistance training is effective in blocking loss of muscle strength. Van Roie et $\mathrm{a}^{24}$ elegantly showed that even a mixed low-resistance exercise protocol in which the resistance training was intensified within a single exercise set $(40 \%$ of 1 repetition maximum) was sufficient to improve muscle strength significantly in older subjects. Despite a large amount of evidence on the significant interaction between cognitive and motoric systems in influencing physical performance in older persons, the current approach is indeed to assess cognitive and physical function in advanced age separately. It is probably time to integrate the motoric and cognitive evaluation, especially in older persons with both cognitive and physical frailty, to draw up specific plans of treatment in patients with balance deficit with primary or secondary sarcopenia. ${ }^{25,26}$ Primary sarcopenia could be treated with an individual plan of physical activity, ${ }^{24}$ while secondary sarcopenia related to, for example, neurological conditions such as dementia or parkinsonism associated with mild cognitive impairment could be particularly influenced by malnutrition with a relatively low level of protein intake. ${ }^{27,28}$ In this regard, epidemiological studies suggested that the international recommendations for the amount of daily protein intake may not be adequate for the maintenance of physical performance in older adults, and some authors have also proposed a dietary intake that includes 25-30 g of high quality protein per meal to obtain a maximal muscle protein synthesis even in older subjects especially during physical exercise. ${ }^{28}$

Our hypothesis is that a "brain-muscle loop" exists in older subjects with some form of cognitive impairment, producing a spiral of muscle loss and subsequent reduction of physical activity with frequent falls and development of mobility-disability. ${ }^{20}$ In fact, recent studies showed that physical frailty is associated with longitudinal decline in global cognitive function in non-demented older adults, ${ }^{29}$ and cognitive impairment could accelerate physical impairment and negative outcomes in older persons. ${ }^{30}$ Further longitudinal studies are necessary to confirm this relationship with a specific plan of treatment for each type of clinical impairment.

When chronic diseases coexist, muscle strength evaluation and cognitive and balance tests should be included in the CGA when observing older persons at risk of falling. ${ }^{31}$ In fact, grip strength seems to be an independent factor associated with mortality in older persons, even all-cause mortality and cardiovascular mortality. ${ }^{32}$ The significance of the prognostic value of the grip strength, if confirmed in other studies, could permit this measure to be considered an independent marker of aging. ${ }^{33}$

The routine use of these assessments could contribute to the development of a prognostic muscle index, taking into account the mixed effect of age-related or disease-related muscle change observed in elderly patients.

\section{Data sharing statement}

The datasets used and/or analyzed during the current study are available from the corresponding author on request. 


\section{Acknowledgment}

The authors wish to thank Mrs Lesley Skeens and Prof Maria Adele Giamberardino for the English revision of the paper.

\section{Author contributions}

All authors (FL, MM, AT, CT, BP, LG, AN, TM) of this manuscript made substantial contributions to the conception and design of the manuscript, were involved in drafting and revising and gave their final approval. All authors contributed toward data analysis, drafting and revising the paper and agree to be accountable for all aspects of the work.

\section{Disclosure}

The authors report no conflicts of interest in this work.

\section{References}

1. Blain H, Masud T, Dargent-Molina P, et al. A comprehensive fracture prevention strategy in older adults: the European Union Geriatric Medicine Society (EUGMS) Statement. J Nutr Health Aging. 2016; 20(6):647-652.

2. Tinetti ME, Speechley M, Ginter SF. Risk factors for falls among elderly persons living in the community. $N$ Engl J Med. 1988;319(26): 1701-1707.

3. Järvinen TL, Michaëlsson K, Jokihaara J, et al. Overdiagnosis of bone fragility in the quest to prevent hip fracture. BMJ. 2015;350:h2088

4. Cameron ID, Gillespie LD, Robertson MC, et al. Interventions for preventing falls in older people in care facilities and hospitals. Cochrane Database Syst Rev. 2012;12:CD005465.

5. El-Khoury F, Cassou B, Latouche A, Aegerter P, Charles MA, Dargent-Molina P. Effectiveness of two year balance training programme on prevention of fall induced injuries in at risk women aged 75-85 living in community: Ossébo randomised controlled trial. BMJ. 2015;351:h3830.

6. Wagner H, Melhus H, Gedeborg R, Pedersen NL, Michaëlsson K. Simply ask them about their balance - future fracture risk in a nationwide cohort study of twins. Am J Epidemiol. 2009;169(2):143-149.

7. Lord SR, March LM, Cameron ID, et al. Differing risk factors for falls in nursing home and intermediate-care residents who can and cannot stand unaided. J Am Geriatr Soc. 2003;51(11):1645-1650.

8. Guralnik JM, Ferrucci L, Simonsick EM, Salive ME, Wallace RB. Lower-extremity function in persons over the age of 70 years as a predictor of subsequent disability. N Engl J Med. 1995;332(9):556-561.

9. Guralnik JM, Simonsick EM, Ferrucci L, et al. A short physical performance battery assessing lower extremity function: association with self-reported disability and prediction of mortality and nursing home admission. J Gerontol. 1994;49(2):M85-M94.

10. Woollacoott MH. Systems contributing to balance disorders in older adults. J Gerontol A Biol Sci Med Sci. 2000;55(8):M424-M428.

11. Shkuratova N, Morris ME, Huxham F. Effects of age on balance control during walking. Arch Phys Med Rehabil. 2004;85(4):582-588.

12. Rantanen T, Guralnik JM, Ferrucci L, et al. Coimpairments as predictors of severe walking disability in older women. $J$ Am Geriatr Soc. 2001;49(1):21-27.

13. Kalyani RR, Corriere M, Ferrucci L. Age-related and disease-related muscle loss: the effect of diabetes, obesity, and other diseases. Lancet Diabetes Endocrinol. 2014;2(10):819-829.

14. Lauretani F, Bautmans I, De Vita F, Nardelli A, Ceda GP, Maggio M. Identification and treatment of older persons with sarcopenia. Aging Male. 2014;17(4):199-204.
15. Mielke MM, Roberts RO, Savica R, et al. Assessing the temporal relationship between cognition and gait: slow gait predicts cognitive decline in the Mayo Clinic Study of Aging. J Gerontol A Biol Sci Med Sci. 2013;68(8):929-937.

16. Ferrucci L, Bandinelli S, Cavazzini C, et al. Neurological examination findings to predict limitations in mobility and falls in older persons without a history of neurological disease. Am J Med. 2004;116(12): 807-815.

17. Lauretani F, Ruffini L, Ticinesi A, Nouvenne A, Maggio M, Meschi T. Accuracy of quantitative positron emission tomography assessment for differentiating cerebral age-related from pathological amyloid deposition: a preliminary report from a case-series study. World J Nucl Med. 2018;17(2):106-111.

18. Savino E, Martini E, Lauretani F, et al. Handgrip strength predicts persistent walking recovery after hip fracture surgery. Am J Med. 2013; 126(12):1068-1075.

19. McLean RR, Shardell MD, Alley DE, et al. Criteria for clinically relevant weakness and low lean mass and their longitudinal association with incident mobility impairment and mortality: the foundation for the National Institutes of Health (FNIH) sarcopenia project. $J$ Gerontol $A$ Biol Sci Med Sci. 2014;69(5):576-583.

20. Lauretani F, Meschi T, Ticinesi A, Maggio M. "Brain-muscle loop" in the fragility of older persons: from pathophysiology to new organizing models. Aging Clin Exp Res. 2017;29(6):1305-1311.

21. Deshpande N, Metter EJ, Lauretani F, Bandinelli S, Ferrucci L. Interpreting fear of falling in the elderly: what do we need to consider? J Geriatr Phys Ther. 2009;32(3):91-96.

22. Beauchet O, Allali G, Annweiler C, Verghese J. Association of motoric cognitive risk syndrome with brain volumes: results from the GAIT study. J Gerontol A Biol Sci Med Sci. 2016;71(8):1081-1088.

23. Arnold P, Bautmans I. The influence of strength training on muscle activation in elderly persons: a systematic review and meta-analysis. Exp Gerontol. 2014;58:58-68.

24. Van Roie E, Delecluse C, Coudyzer W, Boonen S, Bautmans I. Strength training at high versus low external resistance in older adults: effects on muscle volume, muscle strength, and force-velocity characteristics. Exp Gerontol. 2013;48(11):1351-1361.

25. Menant JC, Weber F, Lo J, et al. Strength measures are better than muscle mass measures in predicting health-related outcomes in older people: time to abandon the term sarcopenia? Osteoporos Int. 2017;28(1):59-70.

26. Nyunt MSZ, Soh CY, Gao Q, et al. Characterisation of physical frailty and associated physical and functional impairments in mild cognitive impairment. Front Med (Lausanne). 2017;4:230.

27. Bauer JM, Verlaan S, Bautmans I, et al. Effects of a vitamin D and leucine-enriched whey protein nutritional supplement on measures of sarcopenia in older adults, the PROVIDE study: a randomized, double-blind, placebo-controlled trial. J Am Med Dir Assoc. 2015;16(9): 740-747.

28. Morley JE, Argiles JM, Evans WJ, et al. Nutritional recommendations for the management of sarcopenia. J Am Med Dir Assoc. 2010;11(6): 391-396.

29. Chen S, Honda T, Narazaki K, et al. Physical frailty is associated with longitudinal decline in global cognitive function in non-demented older adults: a prospective study. J Nutr Health Aging. 2018;22(1):82-88.

30. Liu Z, Han L, Gahbauer EA, Allore HG, Gill TM. Joint trajectories of cognition and frailty and associated burden of patient-reported outcomes. J Am Med Dir Assoc. 2018;19(4):304-309.

31. Vieira ER, Palmer RC, Chaves PH. Prevention of falls in older people living in the community. BMJ. 2016;353:i1419.

32. Leong DP, Teo KK, Rangarajan S, et al. Prognostic value of grip strength: findings from the Prospective Urban Rural Epidemiology (PURE) study. Lancet. 2015;386(9990):266-273.

33. Sayer AA, Kirkwood TB. Grip strength and mortality: a biomarker of ageing? Lancet. 2015;386(9990):226-227. 
Clinical Interventions in Aging

\section{Publish your work in this journal}

Clinical Interventions in Aging is an international, peer-reviewed journal focusing on evidence-based reports on the value or lack thereof of treatments intended to prevent or delay the onset of maladaptive correlates of aging in human beings. This journal is indexed on PubMed Central, MedLine,

CAS, Scopus and the Elsevier Bibliographic databases. The manuscript management system is completely online and includes a very quick and fair peer-review system, which is all easy to use. Visit http://www.dovepress. $\mathrm{com} /$ testimonials.php to read real quotes from published authors.

Submit your manuscript here: http://www.dovepress.com/clinical-interventions-in-aging-journal 